\title{
Gibbs Measure as Quantum Ground States
}

\author{
T. Matsui * \\ CNRS Luminy, Case 907, F-13288 Marseille, France
}

Received March 22, 1990

\begin{abstract}
We study certain quantum spin systems which are equivalent to stochastic Ising models. We show that any translationally invariant quantum ground state is given by integration of Gibbs measure. The existence of mass gap is shown to be the same as exponentially fast convergence of stochastic models to invariant states.
\end{abstract}

\section{Introduction}

In [6], we introduce a class of quantum spin systems using translationally invariant ground states. The aim of this paper is to extend our results to a wider class of potentials.

Our new idea is as follows. Assuming certain convergence of finite ground state vectors, we rewrite the Hamiltonian in the form of a generator of a Markov semi-group on the classical spin system.

The ground state property is almost equivalent to the invariance of a measure with respect to the associated Markov semigroup. The proof is rather algebraic. We also prove the existence of the gap of the spectrum of quantum Hamiltonian using results of stochastic Ising models. We use a $C^{*}$ algebraic approach. (See [2] for the basics.)

We first introduce some notations. We consider the algebra of observables $A$ which is the UHF $C^{*}$ algebra,

$$
A=\bigotimes_{\mathbb{Z}^{d}} M_{2}(C) .
$$

So $A$ is generated by Pauli spin matrices $\sigma_{\alpha}^{(j)}(\alpha=x, y, z)$ on $j^{\text {th }}$ site. $\sigma_{\alpha}^{(j)}$ is a selfadjoint unitary satisfying

$$
\begin{aligned}
{\left[\sigma_{\alpha}^{(j)}, \sigma_{\beta}^{(k)}\right] } & =\sigma_{\alpha}^{(j)} \sigma_{\beta}^{(k)}-\sigma_{\beta}^{(k)} \sigma_{\alpha}^{(j)}=0 \\
\sigma_{\alpha}^{(j)} \sigma_{\beta}^{(j)} & =i \varepsilon_{\alpha \beta \gamma} \sigma_{\gamma}^{(j)}
\end{aligned}
$$

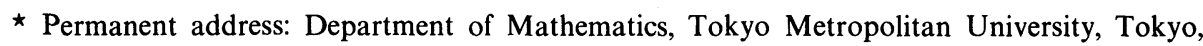
Japan 
where $\varepsilon_{\alpha \beta \gamma}$ is the totally antisymmetric tensor with

$$
\varepsilon_{x y z}=1 .
$$

By $A_{\text {loc }}$, we denote the dense polynomial algebra generated by $\sigma_{\alpha}^{(j)}$. We also define $B$ as the commutative $C^{*}$ algebra generated by $\sigma_{z}^{(j)}$ and we set

$$
B_{\mathrm{loc}}=A_{\mathrm{loc}} \cap B .
$$

Let $X$ be the configuration space of classical Ising spin

$$
X=\{1,-1\}^{\mathbb{Z}^{d}} .
$$

By product topology, $X$ is a compact space. By $\sigma^{(j)}$, we denote the spin variable at the site $j$. Then the algebra of continuous functions on $X$ can be identified with $B$,

$$
\sigma_{z}^{(j)}=\sigma^{(j)}
$$

The quantum spin Hamiltonian we consider is

$$
H=-\sum_{A \neq \varnothing} V_{A}\left(\sigma_{z}\right) \sigma_{x}(A)-\sum_{j \in \mathbb{Z}^{d}} W_{j}\left(\sigma_{z}\right),
$$

where the first sum of (1.5) is taken over all non-empty subsets $A$ in $\mathbb{Z}^{d}$ and

$$
\sigma_{x}(A)=\prod_{j \in A} \sigma_{x}^{(j)}
$$

$V_{A}\left(\sigma_{z}\right)$ and $W_{j}\left(\sigma_{z}\right)$ are in $B_{10 c}$.

Throughout this paper, we make the following assumptions.

\section{Assumption 1.1.}

(i) (Finite rangeness) There exists $r>0$ such that

$$
V_{A}\left(\sigma_{z}\right)=0 \text { for }|A|>r \text { diameter of } A>r,
$$

where $|A|$ is the number of points in $A$.

(ii) The Hamiltonian (1.5) is (lattice) translationally invariant. So we assume that

$$
\begin{aligned}
& \alpha_{k}\left(V_{A}\left(\sigma_{z}\right)\right)=V_{A+k}\left(\rho_{z}\right), \\
& \alpha_{k}\left(W_{j}\left(\sigma_{z}\right)\right)=W_{j+k}\left(\sigma_{z}\right),
\end{aligned}
$$

where $\alpha_{k}$ is an automorphism of $A$ determined by

$$
\alpha_{k}\left(\sigma_{\alpha}^{(j)}\right)=\sigma_{\alpha}^{(j+k)}
$$

for $j, k$ in $\mathbb{Z}^{d} \alpha=x, y, z$.

(iii) $V_{A}\left(\sigma_{z}\right)$ and $W_{j}\left(\sigma_{z}\right)$ are selfadjoint elements of $B_{10 c}$. Furthermore we assume

$$
\begin{aligned}
& V_{A}\left(\sigma_{z}\right) \geqq 0, \\
& V_{A}\left(\sigma_{z}\right)>0 \quad \text { if } \quad|A|=1,
\end{aligned}
$$

and

$$
\left[V_{A}\left(\sigma_{z}\right), \sigma_{x}(A)\right]=0 .
$$

It is well known that (1.5) gives rise to a 1 parameter group of automorphisms of $A$ (see [2]).

$$
\gamma_{t}(Q)=e^{i t H} Q e^{-i t H}
$$


for $t$ in real and $Q$ in $A$. We call $\gamma_{t}()$ the time evolution of quantum system. $A$ state $W$ is $a$ ground state of $\gamma_{t}$ if and only if

$$
\left.\frac{1}{i} \frac{d}{d t} W\left(Q^{*} \gamma_{t}(Q)\right)\right|_{t=0} \geqq 0
$$

for any $Q$ in $A_{\text {loc }}$.

Let $W$ be a ground state and $\left\{\pi_{w}(),. \Omega_{w}, \mathscr{H}_{w}\right\}$ be the G.N.S. triple $\left(\pi_{w}(\right.$.$) the$ representation, $\Omega_{w}$ the cyclic vector and $\mathscr{H}_{w}$ the Hilbert space). Then there exists a selfadjoint operator $H_{w}$ such that

(i) $\quad H_{w} \geqq 0, \quad H_{w} \Omega_{w}=0$,

(ii) $\quad e^{i t H_{w}} \pi_{w}(Q) e^{-i t H_{w}}=\pi_{w}\left(\gamma_{t}(Q)\right)$.

Note that (1.5) does not always converge in ground state representations and we have no explicit construction of $H_{w}$ in general. $H_{w}$ plays a role of the regularized Hamiltonian.

Next we consider classical spins. Any element $f\left(\sigma_{z}\right)$ of $B_{\text {loc }}$ can written as follows:

$$
f\left(\sigma_{z}\right)=\sum_{B} f_{A} \sigma_{z}(B)
$$

Using (1.15) we set (for positive $\delta$ )

where

$$
\|f\|_{\delta}=\sum_{A} e^{\delta\||| A\|}\left|f_{A}\right|,
$$

$$
\text { || } A|\||=|A|+\text { diameter of } A .
$$

If $\delta$ is non-negative, we can prove

$$
\|f\| \leqq\|f\|_{\delta} .
$$

For $f\left(\sigma_{z}\right)$ of (1.15), we also define

$$
f^{(A)}\left(\sigma_{z}\right)=\sum_{|A \cap B|: \text { odd }} f_{B} \sigma_{z}(B) .
$$

Let $\Lambda$ be a finite cube. We consider the quantum Hamiltonian on $\Lambda$. Consider the following equation:

$$
-\sum_{A \cap B \neq \varnothing} V_{A}\left(\sigma_{z}\right) e^{h_{\Lambda}^{(A)}\left(\sigma_{z}\right)}-\sum_{j \in \Lambda} W_{j}\left(\sigma_{z}\right)=E_{\Lambda},
$$

where $V_{A}\left(\sigma_{z}\right)$ and $W_{j}\left(\sigma_{z}\right)$ are ones appeared in (1.5) and $E_{A}$ is ground state energy of the Hamiltonian on $\Lambda$. We impose the periodic boundary condition for $(1.20)$. Then the solution of (1.20) exists in the sense that we can find the unique element $h_{\Lambda}\left(\sigma_{z}\right)$ in $B_{\text {loc }}$ satisfying (1.19) and (1.20). See [6].

Assumption 1.2. We impose the periodic boundary condition for (1.20):

(i) $\left\|h_{\Lambda}^{(B)}\left(\sigma_{z}\right)\right\|_{\delta}$ is bounded uniformly in $\Lambda$ and $B$ for $|B|=1$.

(ii) Let $\Lambda_{l}$ be the hypercube of $\mathbb{Z}^{d}$ centered at the origin with the volume $(21)^{d}$. The following limit exists in the norm \|\|$_{\delta}(\delta>0)$ :

$$
\lim _{l \rightarrow \infty} h_{\Lambda_{l}}^{(B)}\left(\sigma_{z}\right)=h^{(B)}\left(\sigma_{z}\right)
$$

Note that $h_{\Lambda}^{(B)}\left(\sigma_{z}\right)$ is defined via a single function $h_{\Lambda}\left(\sigma_{z}\right)$ and $h^{(B)}\left(\sigma_{z}\right)$ are not 
independent (for different $B$ ). We can define the classical potential $h\left(\sigma_{z}\right)$ as follows:

$$
\begin{aligned}
h^{(B)}\left(\sigma_{z}\right) & =\sum_{|A \cap B| \text { odd }} h_{A} \sigma_{z}(A), \\
h\left(\sigma_{z}\right) & =\sum_{A \subset \mathbb{Z}^{d}} h_{A} \sigma_{z}(A) .
\end{aligned}
$$

By the decay condition (1.16), we can prove the following:

Lemma 1.3. Let $\varepsilon$ be positive. Then there exists $l_{\varepsilon}$ such that if $\Lambda \supset \Lambda_{1}, l>l_{\varepsilon}$,

$$
\begin{gathered}
\sum_{B \ni j}\left|h_{A, B}\right|<\frac{\varepsilon}{3}, \\
\operatorname{dia} B>l_{\varepsilon} \\
\sum_{B \ni j}\left|h_{B}\right|<\frac{\varepsilon}{3}, \\
\operatorname{dia} B>l_{\varepsilon}
\end{gathered}
$$

and

$$
\sum_{B \ni j}\left|h_{A, B}-h_{B}\right|<\varepsilon
$$

if distance $(j, \partial \Lambda)>l_{\varepsilon}$

Proof. (1.23) is a consequence of Assumption 1.2 (i). So we choose $l_{0}$ such that (1.23) is valid. Next we choose $l_{\varepsilon}\left(>l_{0}\right)$ such that

$$
\left\|h_{\Lambda}^{(0)}\left(\sigma_{z}\right)-h^{(0)}\left(\sigma_{z}\right)\right\|<\frac{\varepsilon}{3}
$$

for $\Lambda>\Lambda_{l_{\varepsilon}}$. By translational invariance (or periodicity of boundary conditions) (1.25) implies

$$
\sum_{B \ni j}\left|h_{A, B}-h_{B}\right|<\frac{\varepsilon}{3} .
$$

Thus we have (1.24) if distance $(j, \partial \Lambda)>l_{\varepsilon}$ (q.e.d.).

\section{Remark 1.4.}

(i) The assumption 1.2 can be proved for weakly coupled models by the expansion of [5]. For the Heisenberg model with an external field

$$
H=-\lambda \sum_{j} \sigma_{x}^{(j)}-\sum_{\left|j-j^{\prime}\right|=1}\left(\sigma_{x}^{(j)} \sigma_{x}^{\left(j^{\prime}\right)}+\sigma_{y}^{(j)} \sigma_{y}^{\left(j^{\prime}\right)}+\sigma_{z}^{(j)} \sigma_{z}^{\left(j^{\prime}\right)}\right)
$$

the classical potential $h(\sigma)$ is identically zero. So we have several models of physical interest in our framework.

(ii) In [6], we proved uniqueness of a translationally invariant ground state for the case that $\left\|h^{(j)}\right\|_{\delta}$ is small. We will study the case that $\left\|h^{(j)}\right\|_{\delta}$ is finite, but is large in the rest of this paper. 


\section{2. $L^{2}$ Space of Gibbs Measure}

In this section, we consider the representation of $A$ on the $L^{2}$ space of the Gibbs measure. We assume that readers have some familiarity with the notation of Gibbs measure. For basic facts about Gibbs measure, see [4].

In what follows, the commutative algebra $B$ is regained as the set of continuous functions on $X=\{1,-1\}^{Z^{d}}$. (We use $\sigma^{(j)}$ instead of $\sigma_{z}^{(j)}$.) Let $d \mu(\sigma)$ be a Gibbs measure on $X$ for the potential

$$
h(\sigma)=\sum_{A \subset \mathbb{Z}^{d}} h_{A} \sigma(A),
$$

where $h_{A}$ are determined by (1.21) and (1.22). For a subset $C$ of $\mathbb{Z}^{d}$ and $\sigma$ in $X$, we set

$$
\sigma_{c}=\left\{\begin{array}{ll}
-\sigma^{(j)} & \text { if } j \text { is in } C \\
\sigma^{(j)} & \text { otherwise }
\end{array} .\right.
$$

So $\sigma_{c}$ is the spin flip at $C$.

We now construct the representation of $A$ on $L^{2}(\mu)$. Set

$$
\begin{aligned}
\pi_{\mu}\left(\sigma_{x}^{(j)}\right) F(\sigma) & =\left[\frac{d \mu\left(\sigma_{j}\right)}{d \mu(\sigma)}\right]^{1 / 2} F\left(\sigma_{j}\right), \\
\pi_{\mu}\left(\sigma_{z}^{\left(j^{\prime}\right)}\right) F(\sigma) & =\sigma^{(j)} F(\sigma),
\end{aligned}
$$

where $F(\sigma)$ is in $L^{2}(\mu)$. Note that

$$
\frac{d \mu\left(\sigma_{j}\right)}{d \mu(\sigma)}=\exp \left\{2 \sum_{A \ni j} h_{A} \sigma(A)\right\}
$$

Proposition 2.1. Equation (2.3) gives rise to a representation of the $C^{*}$ algebra $A$ on the Hilbert space of square integrable functions $L^{2}(\mu)$. Furthermore, the von Neumann algebra generated by $\pi_{\mu}\left(\sigma_{z}^{(j)}\right)$ is $L^{\infty}(\mu)$ (acting as multiplication operators) and maimally abelian in $L^{2}(\mu)$.

The above proposition follows directly from the definitions. Let $\Omega_{\mu}$ be the constant function 1. It is cyclic and separating for $\pi_{\mu}(B)^{\prime \prime}=L^{\infty}(\mu)$.

Proposition 2.2. The representation $\pi_{\mu}($.$) of A$ is irreducible if and only if $\mu$ is an extremal Gibbs measure.

Proposition 2.2 was proved for the quantum Potts models in [7] (see Theorem 2.5 of [6]). We note that (2.3) implies

$$
\left(\Omega_{\mu}, \pi_{\mu}\left(\sigma_{z}(A) \sigma_{x}(B) \Omega_{\mu}\right)=\int d \mu(\sigma)\left[\frac{d \mu\left(\sigma_{B}\right)}{d \mu(\sigma)}\right]^{1 / 2} \sigma(A) .\right.
$$

The next task is to construct a regularized Hamiltonian $H_{\mu}$ on $L^{2}(\mu)$. Let $\Lambda$ be a cube in $\mathbb{Z}^{d}$, and we set

$$
\tilde{H}_{A}=-\sum_{A \cap \Lambda \neq \varnothing} V_{A}\left(\sigma_{z}\right)\left\{\sigma_{z}(A)-e^{h^{(A)}}\left(\sigma_{z}\right)\right\},
$$

where $h^{(A)}\left(\sigma_{z}\right)$ is defined in (1.21). 
Proposition 2.3. The following limit exists in the sense of strong resolvent convergence:

$$
\lim _{\Lambda \rightarrow \infty} \pi_{\mu}\left(\tilde{H}_{\Lambda}\right)=H_{\mu}
$$

Furthermore $\pi_{\mu}\left(A_{\mathrm{loc}}\right) \Omega_{\mu}$ is a core for $H_{\mu}$ and $H_{\mu}$ is positive,

$$
\begin{aligned}
e^{i t H_{\mu}} \pi_{\mu}(Q) e^{-i t H_{\mu}} & =\pi_{\mu}\left(\gamma_{t}(Q)\right), \\
H_{\mu} \Omega_{\mu} & =0 .
\end{aligned}
$$

Proof. We first show that

$$
\lim _{\Lambda \rightarrow \infty}\left[\tilde{H}_{\Lambda} Q\right]=[H, Q]
$$

for $Q$ in $A_{\text {loc }}$. In view of (1.20), we have

$$
[H, Q]=\left[\tilde{H}_{\Lambda}, Q\right]=-\sum_{A \cap \Lambda \neq \varnothing}\left[\left(e^{h_{\Lambda}^{(A)}\left(\sigma_{z}\right)}-e^{h^{(A)}\left(\sigma_{z}\right)}\right) V_{A}\left(\sigma_{z}\right), Q\right] .
$$

for sufficiently large $\Lambda$. Here we used the fact that $Q$ is strictly local element, and $H$ is of finite range. We consider $Q=\sigma_{x}^{(0)}$. For simplicity of exposition, we assume

$$
\begin{array}{lll}
V_{A}\left(\sigma_{z}\right)=0 & \text { if } & |A|>1, \\
V_{A}\left(\sigma_{z}\right)=1 & \text { if } & |A|=1 .
\end{array}
$$

It suffices to estimate

$$
\sum_{j \in \Lambda}\left[\left(e^{h_{\Lambda}^{(j)}\left(\sigma_{z}\right)}-e^{h^{(j)}\left(\sigma_{z}\right)}\right) \sigma_{x}^{(0)}\right] .
$$

Each summand of (2.13) is bounded by

$$
\left\|\left\{e^{h_{\Lambda}^{(j)}\left(\sigma_{0}\right)}-e^{h^{(j)}\left(\sigma_{0}\right)}\right\}-\left\{e^{h_{\Lambda}^{(j)}(\sigma)}-e^{h^{(j)}(\sigma)}\right\}\right\| .
$$

We also use

$$
e^{h^{(j)}\left(\sigma_{0}\right)}-e^{h^{(j)}(\sigma)}=\cosh \left(\frac{h^{(j)}\left(\sigma_{0}\right)+h^{(j)}(\sigma)}{2}\right) \operatorname{sink}\left(\frac{h^{(j)}\left(\sigma_{0}\right)-h(\sigma)}{2}\right) .
$$

Thus (2.13) is bounded by the following two terms:

$$
\begin{aligned}
& \sum_{j \in \Lambda}\left\|\cosh \left(\frac{h_{\Lambda}^{(j)}\left(\sigma_{0}\right)+h_{\Lambda}^{(j)}(\sigma)}{2}\right)-\cosh \left(\frac{h^{(j)}\left(\sigma_{0}\right)+h^{(j)}(\sigma)}{2}\right)\right\| \\
& \|\times\| \operatorname{sink}\left(\frac{h_{\Lambda}^{(j)}\left(\sigma_{0}\right)-h_{\Lambda}^{(j)}(\sigma)}{2}\right) \|, \\
& \sum_{j \in \Lambda}\left\|\cosh \left(\frac{h^{(j)}\left(\sigma_{0}\right)+h^{(j)}(\sigma)}{2}\right)\right\| \times \| \operatorname{sink}\left(\frac{h_{\Lambda}^{(j)}\left(\sigma_{0}\right)-h_{\Lambda}^{(j)}(\sigma)}{2}\right) \\
& \quad-\left(\frac{h^{(j)}\left(\sigma_{0}\right)-h^{(j)}(\sigma)}{2}\right) \| .
\end{aligned}
$$

By the boundedness assumption of $h_{\Lambda}^{(j)}(\sigma)$, we have

$$
(2.14 \mathrm{~b}) \leqq C_{1} \sum_{j \in \Lambda}\left\|\frac{h_{\Lambda}^{(j)}\left(\sigma_{0}\right)-h_{\Lambda}^{(j)}(\sigma)}{2}-\frac{h^{(j)}\left(\sigma_{0}\right)-h^{(j)}(\sigma)}{2}\right\|
$$




$$
\begin{aligned}
& \leqq C_{1} \sum_{j \in \Lambda} \sum_{A \ni j, 0}\left|h_{\Lambda, A}-h_{A}\right| \\
& \leqq C_{1} \sum_{A \ni 0}|A| h_{\Lambda, A}-h_{A} \mid \leqq \frac{C_{1}}{\delta}\left\|h_{\Lambda}^{(0)}(\sigma)-h^{(0)}(\sigma)\right\|_{\delta} .
\end{aligned}
$$

In $(2.14 a)$ we split the sum as

$$
\sum_{|j-\partial \Lambda|>l_{\varepsilon}}+\sum_{|j-\partial \Lambda|<l_{\varepsilon}}
$$

In the first term, we apply (1.24), so

$$
\begin{aligned}
\sum_{|j-\partial \Lambda|>l_{\varepsilon}} \cdots & \leqq C_{2} \varepsilon \sum_{j \in \Lambda}\left\|\frac{h_{\Lambda}^{(j)}\left(\sigma_{0}\right)-h_{\Lambda}^{(j)}(\sigma)}{2}\right\| \\
& =C_{2} \varepsilon \sum_{j \in \Lambda} \sum_{j_{0} \in \Lambda}\left|h_{\Lambda, A}\right| \\
& \leqq C_{2} \varepsilon\left\|h_{\Lambda}^{(0)}(\sigma)\right\|_{0} .
\end{aligned}
$$

In the second term of (2.15), we use decay of potential. So

$$
\begin{aligned}
\sum_{|j-\partial \Lambda|<l_{\varepsilon}} \cdots & \leqq C_{3}{ }^{\prime} \sum_{|j-\partial \Lambda|<l_{\varepsilon}}\left\|h_{\Lambda}^{(j)}\left(\sigma_{0}\right)-h_{\Lambda}^{(j)}(\sigma)\right\| \\
& \leqq C_{3} \sum_{|j-\partial \Lambda|<l_{\varepsilon}} \sum_{A \ni j, 0}\left|h_{\Lambda, A}\right| \\
& \leqq C_{4} \sum_{|j-\partial \Lambda|<l_{\varepsilon}} e^{-\delta|j|}
\end{aligned}
$$

So (2.17) goes to zero as $\Lambda$ goes to $\mathbb{Z}^{d}$. Thus we have (2.10). The claim of the proposition follows from (2.10) and the fact that $A_{\text {loc }}$ is a dense subset of analytic elements of $\gamma_{t}($.$) , and$

$$
\tilde{H}_{\Lambda} \Omega_{\Lambda}=0 \text {. }
$$

See Theorem 6.2.4 of [1] and Theorem VIII, 25. of $e$ [8]. (q.e.d.)

Remark 2.4. Observant reads may realize that (2.6) is the generator of a Markov semigroup $S(t)$ on $C(X)$. It is possible to prove that the following equation gives rise to a Markov generator (see [4]),

$$
\lim _{\Lambda \rightarrow \infty} \pi_{\mu}\left(\tilde{H}_{\Lambda}\right) \pi_{\mu}(Q) \Omega_{\mu}=\pi_{\mu}([H, Q]) \Omega_{\mu}
$$

for $Q$ in $B_{\text {loc }}$. We will return to this observation later.

\section{Main Results}

Let $\mu$ be a Gibbs measure and $W_{\mu}$ be the vector state associated to $\Omega_{\mu}$ of the preceding section. We can now state our main results of this paper.

Let $\Lambda$ be a cube of $\mathbb{Z}^{d}$

$$
H_{\Lambda}=-\sum_{\Lambda \cap A \neq \varnothing} V_{A}\left(\sigma_{z}\right) \sigma_{z}(A)-\sum_{j \in \Lambda} W_{j}\left(\sigma_{z}\right)
$$

where we use the periodic boundary condition. Let $E_{\boldsymbol{A}}$ be the smallest eigenvalue of $H_{\Lambda}$. 
Theorem 3.1. Suppose a state $W$ satisfies the following:

$$
W\left(\tilde{H}_{\Lambda}\right)=0
$$

for any finite $\Lambda$.

(i) $W$ is a ground state of $\gamma_{t}$ (in the sense of (1.12)) and there exists a Gibbs measure $\mu$ for $h(\sigma)$ in (1.22) such that

$$
W\left(\sigma_{z}(A) \sigma_{x}(B)\right)=\int d \mu(\sigma) \sigma(A)\left[\frac{d \mu\left(\sigma_{B}\right)}{d \mu(\sigma)}\right]^{1 / 2} .
$$

The G.N.S. representation associated with $W$ is equivalent to (2.3),

(ii) Let $H_{W}$ be the selfadjoint operator defined by (2.7). Then $H_{W}$ satisfies (1.13) and (1.14).

(iii) $W$ is a pure ground state if and only if $\mu$ is an extremal Gibbs measure of $X$.

Corollary 3.2. Let $W$ be a translationally invariant ground state, then there exists a translationally invariant Gibbs measure $\mu$ such that all the conclusions of Theorem 3.1 are valid.

\section{Remark 3.3.}

(i) The above results are valid if we use free boundary conditions.

(ii) Corollary 3.2 should be compared with Theorem 5.12 of [4].

Next consider the case that the Gibbs measure is unique. Let $L$ be the generator of the Stochastic Ising model determined by

$$
L=\sum_{j \in \mathbf{Z}_{d}} V_{\{j\}}\left(\sigma_{z}\right)\left\{\pi_{\mu}\left(\sigma_{x}^{(j)}\right)-\pi_{\mu}\left(e^{h^{(j)}}\left(\sigma_{z}\right)\right)\right\}
$$

Theorem 3.4. Suppose that the Gibbs measure for $h(\sigma)$ is unique. Suppose further that the $L^{2}$ spectrum of the (unique) selfadjoint extension of $L$ in (3.4) has a gap $\varepsilon$ above zero eigenvalue, i.e. $\operatorname{spec}(L) \cap(0, \varepsilon)=\varnothing$. Then the spectrum of $H_{\mu}$ has a gap larger than $\varepsilon$ above zero eigenvalue. The zero eigenvalue is of multiplicity one.

Remark 3.5. We now have many results on the gap of spectrum of $L$. (See $[4,3]$ and references therein.) Theorem 3.4 implies the existence of gap for weakly coupled (= high temperature) models treated in [6].

We can also establish the existence of gap for highly anisotropic Heisenberg model of spin $1 / 2$ (without the external field term). This means that

$$
\begin{array}{lll}
V_{A}\left(\sigma_{z}\right)=0 & \text { if } & |A| \neq 2, \\
V_{A}\left(\sigma_{z}\right)>0 & \text { if } & |A|=2,
\end{array}
$$

in the notation (1.5). The ground site is doubly degenerated and it causes some complications. We will explain this in a separate paper.

\section{Proofs}

We give a proof of results stated in Sect. 3. Let $A$ be a subset of $\mathbb{Z}^{d}$. We set

$$
C_{A}=\frac{1}{2}\left\{\mathbb{1}-\sigma_{x}(A)\right\} e^{(1 / 2) h^{(A)}\left(\sigma_{z}\right)} \text {. }
$$




\section{Lemma 4.1.}

$$
\frac{1}{2} C_{A}^{*} V_{A}\left(\sigma_{z}\right) C_{A}=(-1) V_{A}\left(\sigma_{z}\right)\left\{\sigma_{x}(A)-e^{h^{(A)}\left(\sigma_{z}\right)}\right\}
$$

Proof. Note that

$$
\sigma_{x}(A) e^{(1 / 2) h^{(A)}\left(\sigma_{z}\right)}=e^{-(1 / 2) h^{(A)}\left(\sigma_{z}\right)} \sigma_{x}(A),
$$

this follows from the definition of $h^{(A)}\left(\sigma_{z}\right)$. Then (1.10) and (4.3) lead to (4.2). (q.e.d.)

Lemma 4.2. Equation (3.2) implies the following

$$
W\left(C_{A}^{*} C_{A}\right)=0
$$

for any $A$ with $|A|=1$.

Proof. By Lemma 4.2, (3.2) leads to

$$
\left.\sum_{A} W\left(C_{A}^{*} V_{A}\left(\sigma_{z}\right) C_{A}\right)=0\right)
$$

By our assumption (1.9), we get (4.8).

Poof of Theorem 3.1. Let $W$ be a state of $A$ satisfying (3.2). Let $\left\{\pi_{W}(),. \Omega_{W}, \mathscr{H}_{W}\right\}$ be the G.N.S. triple for $W$. Then Lemma 4.2 and (4.1) (4.3) imply

$$
\begin{aligned}
& \pi_{W}\left(C_{(j)}\right) \Omega_{W}=0, \\
& \pi_{W}\left(\sigma_{x}^{(j)}\right) \Omega_{W}=\pi_{W}\left(e^{h^{(j)}\left(\sigma_{z}\right)}\right) \Omega_{W} .
\end{aligned}
$$

Let $\mu$ be the measure on $X$ defined by

$$
\int d \mu(\sigma) \sigma(A)=W\left(\sigma_{z}(A)\right) .
$$

The adjoint action $\operatorname{Ad}\left(\sigma_{x}^{(j)}\right)$ implements the spin flip at $j$ on $C(X)$. So (4.6) may read

$$
\frac{d \mu\left(\sigma_{j}\right)}{d \mu(\sigma)}=e^{2 h^{(j)}(\sigma)}
$$

Due to Lemma 2.1 of [9], $\mu$ is a Gibbs measure for $h(\sigma)$. (The measure satisfying (4.11) is known to be a solution of the DLR equation.) So we have (1). Other statements of the theorem follows from results of Sect. 2. (q.e.d.)

Corollary 3.2 follows from Lemma 4.3, Theorem 3.1 and a result of [1], namely, if $W$ is a translationally invariant ground state, then

$$
\lim _{l \rightarrow \infty} \frac{1}{\left|\Lambda_{l}\right|} W\left(H_{\Lambda_{l}}-E_{\Lambda_{l}}\right)=0 .
$$

\section{Lemma 4.3.}

$$
\lim _{l \rightarrow \infty} \frac{1}{\left|\Lambda_{l}\right|}\left\|\left(H_{\Lambda_{l}}-E_{\Lambda_{l}}\right)-\tilde{H}_{\Lambda_{l}}\right\|=0 .
$$

Proof. For simplicity, we again assume (2.12). By (1.20)

$$
\left(H_{\Lambda_{l}}-E_{\Lambda_{l}}\right)-\tilde{H}_{\Lambda_{l}}=\sum_{j \in \Lambda_{l}}\left\{e^{h_{\lambda_{l}}^{(j)}\left(\sigma_{z}\right)}-e^{h^{(j)^{\prime}}\left(\sigma_{z}\right)}\right\}
$$


So using Lemma 1.3 we have.

$$
\frac{\|(4.11)\|}{\left|\Lambda_{l}\right|} \leqq\left(\sum_{|j-\partial \Lambda|<l_{\varepsilon}} \mid\right) \frac{C}{\left|\Lambda_{l}\right|}+\varepsilon .
$$

We now turn to Theorem 3.4. (q.e.d.)

\section{Lemma 4.4.}

(i) Let $W$ be a pure ground state of $\gamma_{t}$. The selfadjoint operator satisfying (1.13) and (1.14) is unique.

(ii) Suppose that $W$ is a pure ground state satisfying

$$
W\left(A^{*} \delta(A)\right) \geqq \varepsilon\left(W\left(A^{*} A\right)-|W(A)|^{2}\right)
$$

for any $A$ in $A_{\text {loc }}$ and

$$
\delta(A)=[H, A] .
$$

Then the unique selfadjoint operator $H_{W}$ of (1.13) and (1.14) has a gap of size $\varepsilon$ in the spectrum above zero. The zero eigenvalue is of multiplicity one.

Proof of Lemma 4.4. Let $\left\{\pi_{W}(),. \Omega_{W}, \mathscr{H}_{W}\right\}$ be the G.N.S. triple of $W$. It follows from irreducibility of $\pi_{W}($.$) . Next recall that \pi_{W}\left(A_{1 \mathrm{loc}}\right) \Omega_{W}$ is core for $H_{W}$ (elements of $A_{\text {loc }}$ are analytic for $\left.\gamma_{t}().\right)$, see [2]. Equation (4.13) is equivalent to

$$
\left(\xi_{A}, H_{W} \xi_{A}\right) \geqq \varepsilon\left(\xi_{A}, \xi_{A}\right)
$$

where

$$
\xi_{A}=\pi_{W}(A) \Omega_{W}-\left(\Omega_{W}, \pi_{W}(A) \Omega_{W}\right) \times \Omega_{W} .
$$

(Note $\left.W\left(A^{*} \delta(A)\right)=\left(\pi_{W}(A) \Omega_{W}, H_{W} \pi_{W}(A) \Omega_{W}\right)\right)$. As $\pi_{W}\left(A_{\text {loc }}\right) \Omega_{W}$ is core, (4.15) says that the spectrum of $H_{W}$ is larger than $\varepsilon$ in the orthogonal complement of $\Omega_{W}$. So we have the conclusion. (q.e.d.)

Proof of Theorem 3.4. Let $W$ and $\mu$ be as in Theorem 3.4. Then first we have for any $A$ in $\mathbb{Z}^{d}$,

$$
W\left(C_{A}^{*} C_{A}\right)=0 .
$$

This is due to (2.3) and (2.4). Thus

$$
\begin{aligned}
W\left(Q^{*} \delta(Q)\right) & =\lim _{\Lambda \rightarrow \infty} \sum_{A \cap A \neq \varnothing} W\left(Q^{*}\left[C_{A}^{*} V_{A}\left(\sigma_{z}\right) C_{A}, Q\right]\right) \\
& =\sum_{A} W\left(Q^{*} C_{A}^{*} V_{A}\left(\sigma_{z}\right) C_{A} Q\right) \\
& \geqq \sum_{|A|=1} W\left(Q^{*} C_{A}^{*} V_{A}\left(\sigma_{z}\right) C_{A} Q\right)=W\left(Q^{*} \tilde{L} Q\right)
\end{aligned}
$$

for any $Q$ in $A_{\text {loc }}$. Here $\tilde{L}$ is the selfadjoint extension of $L$ in (3.4). If $\tilde{L}$ has a gap $\varepsilon$ of spectrum above zero eigenvalue it is easy to show

$$
\begin{aligned}
\left(\pi_{W}(Q) \Omega_{W}, \tilde{L} \pi_{W}(Q) \Omega_{W}\right) & \geqq \varepsilon\left\{\left\|\pi_{W}(Q) \Omega_{W}\right\|^{2}-\left\|\left(\Omega_{W} \pi_{W}(Q) \Omega_{W}\right)\right\|^{2}\right. \\
& =\varepsilon\left\{W\left(Q^{*} Q\right)-|W(Q)|^{2}\right\} .
\end{aligned}
$$

Due to (4.18) and Lemma 4.4, we have Theorem 3.4. (q.e.d.) 
Acknowledgements. This work was done during the author's stay at the University of Leuven and Centre de Physique Théorique, CNRS Luminy, France. The author would like to thank them for their financial support and hospitality. The author benefited greatly from many discussions with Prof. A. Verbeure and Dr. C. Maes.

\section{References}

1. Bratteli, O., Kishimoto, A., Robinson, D.: Ground states of quantum spin systems. Commun. Math. Phys. 64, 41-48 (1978)

2. Bratteli, O., Robinson, D.: Operator algebras and quantum statistical mechanics. II. Berlin, Heidelberg, New York: Springer 1981

3. Holley, R.: Rapid convergence to equilibrium in one dimensional stochastic Ising model. Ann. Prob. 13, 72-89 (1985)

4. Kirkwood, J. R., Thomas, L.: Expansion and phase transitions for ground states of quantum Ising lattice systems. Commun. Math. Phys. 88, 569-580 (1983)

5. Liggett, T.M.: Interacting particle systems. Berlin, Heidelberg, New York: Springer 1985

6. Matsui, T.: Uniqueness of the translationally invariant ground state in quantum spin systems. Commun. Math. Phys. 126, 453-467 (1990)

7. Matsui, T.: A link between quantum and classical Potts models. J. Stat. Phys. (to appear)

8. Reed, M., Simon, B.: Methods of mathematical physics (II). New York: Academic Press

9. Shiga, T.: Z. Wahrsch. Theor. Verw. Geb 39, 339 (1977)

10. Thomas, L.: Quantum Heisenberg feromagnets and Stochastic exclusion process. J. Math. Phys. 21, 1921-1924 (1980)

Communicated by H. Araki 
